Plant Molecular Biology 3: 127-136 (1984).

(C) Martinus Nijhoff/DrW. Junk Publishers, The Hague. Printed in the Netherlands.

\title{
Structure and rearrangements of rRNA genes in chloroplast DNA in two strains of Euglena gracilis
}

\author{
Barbara Koller ${ }^{1}$, Hajo Delius ${ }^{1} \&$ Robert B. Helling 2,3 \\ ${ }^{1}$ European Molecular Biology Laboratory, 69 Heidelberg, F.R.G. \\ 2 Institut de Biologie Moléculaire et Cellulaire, 15 rue Descartes, 67084 Strasbourg, France \\ ${ }^{3}$ Permanent address: Division of Biological Sciences, University of Michigan, Ann Arbor, MI 481009, \\ U.S.A.
}

Keywords: Euglena gracilis, strain bacillaris, strain Z-S, chloroplast, rRNA genes

\begin{abstract}
Summary
The organisation of the rRNA genes in the chloroplast genomes of two strains of Euglena gracilis were analyzed and compared. It was previously shown that the bacillaris strain contains three complete rrn (rRNA) operons (7) and that the Z-S strain contains one operon (21). Using heteroduplex analysis it was found that the bacillaris strain contains, apart from the three complete rrn operons, an extra 16S rRNA gene, an extra partial 23S rRNA gene sequence and an inverted duplication of a stretch within the $5 \mathrm{~S}-16 \mathrm{~S}$ spacer. In addition a short $(<100 \mathrm{bp}$ ) inverted repeat sequence (I3) which forms a stem/loop structure in singlestranded cpDNA was located between the 3'-end of the extra 16S rRNA gene and the partial $23 \mathrm{~S}$ rRNA sequence.

The Z-S strain differs from the bacillaris strain by a deletion of two units of the complete rrn operons. The region upstream of the single complete rrn operon, including the inverted repeats, the partial $23 \mathrm{~S}$ and the extra 16S rRNA sequences is identical with the bacillaris strain.

The only non-homology found in heteroduplexes between the Sall fragments of B of the two strains is the deletion-insertion loop which represents the two rrn operons. A small deletion loop was found occasionally in hetero- and in homoduplexes of both strands in the region of variable size. Apart from the deletion/insertion of two rrn operons the two genomes appear to be colinear as can be seen from partial denaturation mapping. The organisation of the rRNA genes of the two strains is compared with those of the $\mathrm{Z}$ strain and the bacillaris-ATCC strain.
\end{abstract}

\section{Introduction}

The chloroplast genomes of different strains of Euglena gracilis contain a variable number of tandemly repeated rrn operons. Strain $\mathrm{Z}$ and strain bacillaris $(16,7)$ contain three operons, whereas the strains Z-S and bacillaris-ATCC contain one and five operons, respectively $(21,13)$. In addition the presence of one, and of two extra 16S rRNA genes was demonstrated for the $\mathrm{Z}$ and for the bacillarisATCC strains, respectively $(9,13)$. The structures of the rRNA gene sets in the latter two strains seem to be related by a simple deletion or insertion of a fragment containing two complete rrn operons and one extra 16S rRNA gene. In both cases the extra $16 \mathrm{~S}$ rR NA gene is located near an inverted repeat structure $(11,13)$. A sequence of $28 \mathrm{bp}$ from the 5 -terminal part of the structural 16S rRNA sequence was found to be inserted into the leader sequence of this gene in an inverted orientation. $\mathrm{SI}$-endonuclease protection analysis suggests that the extra 16S rRNA gene in the $Z$ strain is not transcribed into a stable rRNA (17).

It will be interesting to know whether the extra $16 \mathrm{~S}$ rRNA gene plays an essential role in the chloroplast genome or instead represents the non-functional product of recombination and genetic drift. To find out whether the extra 16S rRNA gene also 
occurs in other Euglena strains, the bacillaris and the Z-S strain were analysed by electron microscopy. To determine the organization of the rRNA genes in these two strains, the cpDNAs were isolated and hybridized with the $E$. coli rrnD operon and with a homologous fragment containing the $\operatorname{rrnA}$ operon of the bacillaris strain. The two strains were also compared directly by heteroduplex analysis and partial denaturation mapping.

\section{Materials and methods}

Euglena gracilis strain bacillaris was obtained from J. Schiff through W. E. Barnett and has been described, (7). Euglena gracilis strain Z-S was obtained from D. E. Buetow (21). Cultivation of strains and extraction of chloroplast DNA (cpDNA) was done as described before for the $\mathrm{Z}$ strain (11). The construction of the plasmids pBK8 (2) and pRBH26 (5), and the conditions for heteroduplex preparation (3) were described before. The pBK8 DNA was a gift from Ibolya Kiss (Szeged). The DNA of a plasmid which contained the region between the extra 16S rRNA gene and the rrnA operon of the Euglena gracilis strain $\mathrm{Z}$ was a gift from E. Roux (Neuchatel).

For the partial denaturation of the cpDNA high $\mathrm{pH}$ conditions were used as described by Inman \& Schnoes (8). After the denaturation the samples were transferred into $10 \mathrm{mM}$ Tris- $\mathrm{HCl}, \mathrm{pH} 7.4$, $1 \mathrm{mM}$ EDTA by passage through a column with Sepharose CL-2B. Samples were spread with cytochrome c from $30 \%$ formamide, $0.1 \mathrm{M}$ Tris- $\mathrm{HCl}$, pH 8.5, 1 mM EDTA onto a hypophase of $0.005 \%$ octyl glucopyranoside (Sigma).

Length measurements of the BamHI fragments $\mathrm{E}, \mathrm{D}$ and $\mathrm{F}$ from the bacillaris strain, were done with isolated double-stranded fragments adsorbed to mica, using PM2 DNA as an internal length standard, following the method used for Vicia cpDNA $(3,19)$. Length measurements of circular cpDNA were done in cytochrome spreadings using T7 DNA as internal length standard (4).

\section{Results}

The molecular sizes of the cpDNA of the bacillaris strain and the Z-S strain were measured on double-stranded intact circular molecules, using T7 DNA as length standard (4). The bacillaris strain has a size of $145 \pm 2 \mathrm{kbp}$ and the Z-S strain a size of $133 \pm 2 \mathrm{kbp}$.

The single-stranded circular cpDNA molecules of Euglena gracilis strain bacillaris display two inverted repeat structures in cytochrome spreadings (see Fig. 1). One inverted repeat of less than $100 \mathrm{bp}$ forms a stem/loop structure which was designated $\mathrm{I} 3$, in analogy to a similar structure found previously in the $\mathrm{Z}$ strain and in the bacillaris-ATCC strain $(11,13)$. The other inverted repeat has a size of about $300 \mathrm{bp}$, and forms a small loop. It is designated the hairpin.

To visualize the rRNA genes the cpDNA was reannealed with an $E$. coli fragment which contains the rrnD operon. The $E$. coli BamHI fragment was cut out from the plasmid pBK8 (2) and purified by elution from an agarose gel after electrophoresis. Fig. 1 shows heteroduplexes between cpDNA and the $E$. coli $\mathrm{rrnD}$ operon. The short single-stranded tail of the $E$. coli fragment indicates the position of the 5 '-end of the $16 \mathrm{~S}$ rRNA sequence, the long tail the non-homology downstream of the 3'-end of the 23S rRNA genes. The positions of the genes and the direction of transcription can be deduced from these heteroduplex structures.

Heteroduplexes with the three complete operons were observed downstream of the hairpin structure. The three operons $\operatorname{rrnA}, \operatorname{rrnB}$, and $\operatorname{rrnC}$ could be identified by their distance from the hairpin. Figure la shows heteroduplexes with the first two operons. In some cases the $E$. coli fragment annealed with a 16S rRNA gene upstream of the hairpin and the stem/loop and with the 23S rRNA gene of the rrnA operon (Fig. 1b). This demonstrates that the bacillaris strain also has an extra $16 \mathrm{~S}$ rRNA sequence which is not part of a complete operon. The hairpin and the stem/loop I3 are located between this gene and the 16S rRNA gene of the first operon. The positions of the rRNA genes in relation to the inverted repeats were measured in these heteroduplexes and are drawn in the map in Fig. 5.

The leader sequence upstream of the 16S rRNA gene and the sequence downstream of the 23S rRNA gene including the 5S rRNA gene of $E$. coli do not hybridize with the corresponding chloroplast regions. To see whether the extra 16S rRNA gene is flanked on either side by a leader sequence 

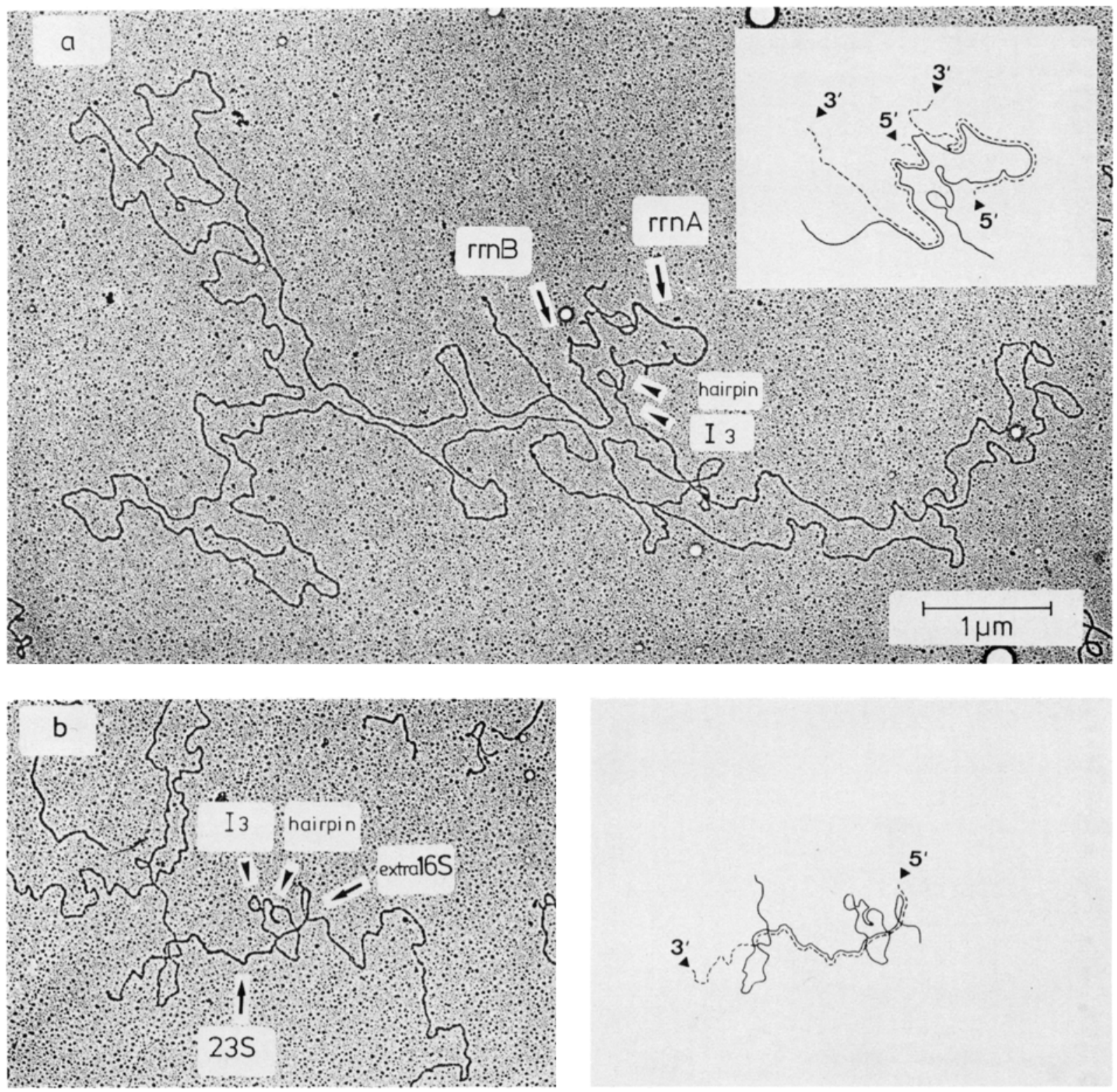

Fig. 1. Electron micrographs of heteroduplexes between Euglena gracilis strain bacillaris cpDNA and the BamHI fragment from the plasmid $\mathrm{pBK} 8$ which contains the $E$. coli $\mathrm{rrnD}$ operon (2).

a) Two $E$. coli fragments have annealed with the first ( $\operatorname{rnA}$ ) and the second ( $\mathrm{rnB} B$ RNA operons. A stem/loop structure termed 13 which was formed by a short inverted repeat $(<100 \mathrm{bp})$ and a hairpin which was formed by an inverted repeat of about $300-400 \mathrm{bp}$ were observed in the single-stranded cpDNA upstrea $m$ of the first rrn operon. In the tracing the cpDNA is drawn as a solid line and the $E$. col $i$ fragment as a dashed line. The 5 'end of the $E$. coli $16 \mathrm{~S}$ rRNA gene is positioned about $300 \mathrm{bp}$ away from the end of the fragment and is indicated (marked 5'). The long single-stranded end is the region downstream of the 3'-end of the E. coli $23 \mathrm{~S}$ rRNA gene (marked 3').

b) One $E$. coll fragment was hybridized with an extra $16 \mathrm{~S}$ rRNA gene which is located upstream of the inverted repeats. The heteroduplex continues with the 23S rRNA gene of the rrnA operon. The single-stranded loop between these duplex regions contains the stem/loop 13, the hairpin, and the 16S rRNA gene of the rmA operon. 

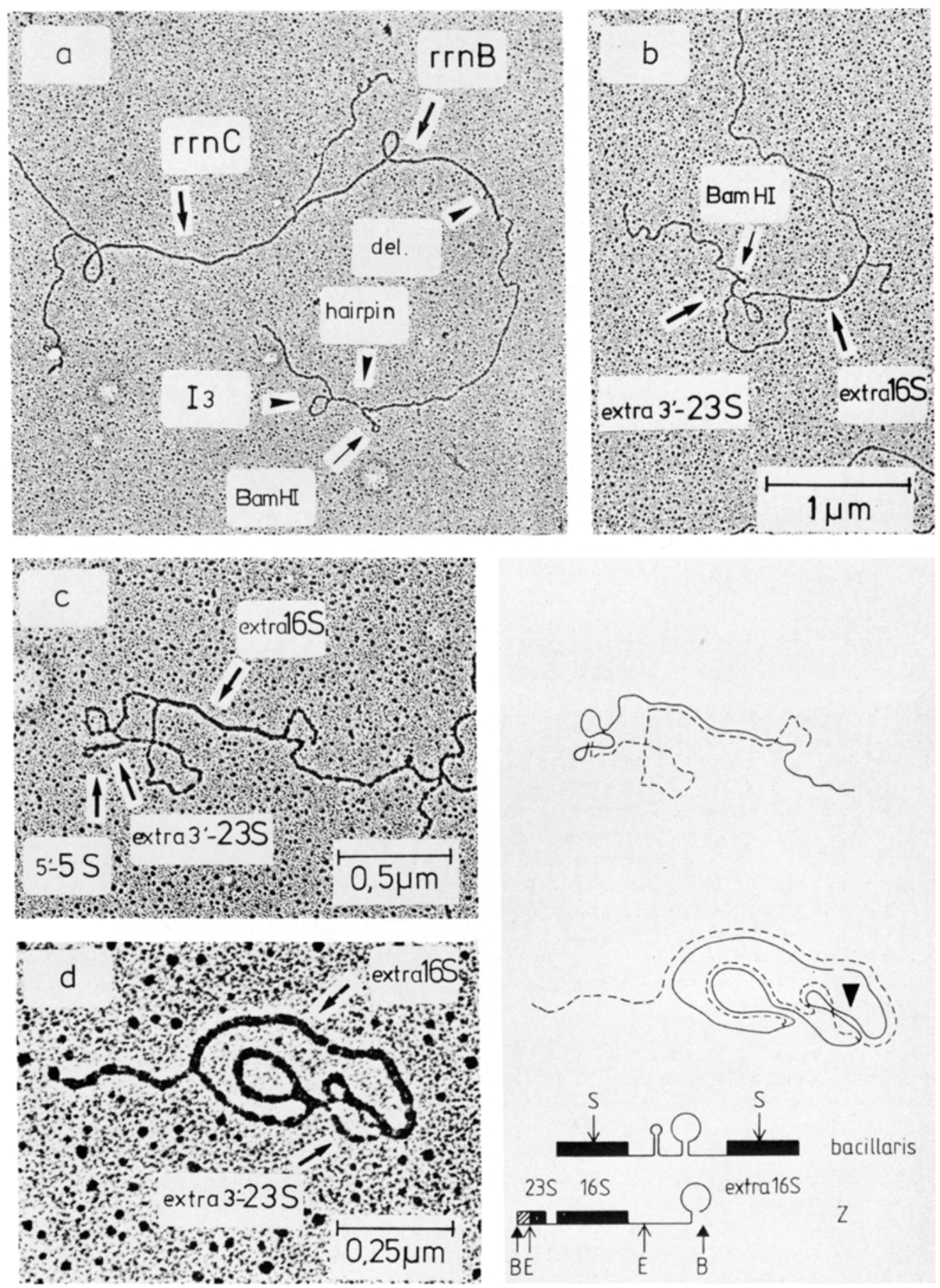
or other parts of the complete rrn operons, the cpDNA was hybridized with the plasmid pRBH26, which contains the BamHI E fragment (the rrnA operon), cloned in pBR322 from the same Euglena strain (5). The BamHI cleavage site in cpDNA is at position +26 in each of the four 5S rRNA genes. The BamHI E fragment therefore contains about $100 \mathrm{bp}$ of the first 5S rRNA gene on one end and 26 bp of the second SS rRNA gene at the other end (6). The restriction enzyme SalI has one site in the vector sequence but none in the insert. Digestion of pRBH26 DNA with Sall produces a linear molecule with about 200 bp of the vector sequence at the 5 '-end of the rrnA operon and the remainder of pBR322 at the 3'-end of the operon. Fig. 2a shows a heteroduplex between the linearized pR BH26 DNA and a random cpDNA fragment. The short knob and the long single-stranded tail of the vector serve as a marker for the orientation of the rrn operons. It has been shown previously that the spacer between operons A and B has a 292 bp deletion as compared with the corresponding sequence before operons $A$ and $C(6)$. A small deletion loop is displayed at the expected position.

Reannealing of the plasmid DNA with the rrnA operon or with the extra 16S rRNA gene resulted in very complicated structures. This was due to the hairpin and other repeated sequences, as will be described below. The distance between the first 5S rRNA gene and the 5'-end of the 16S rRNA gene of operon rrnA has been determined by sequencing (6). The position of the hairpin in relation to the 5 '-end of this $16 \mathrm{~S}$ rRNA gene has been measured in heteroduplexes described in Fig. 1. It can thus be calculated that the first 5S rRNA gene must be positioned within the small loop of the hairpin. Digestion of cpDNA with BamHI must therefore separate the two inverted repeat sequences of the hairpin. The left sequence should be in the BamHI $E$ fragment and the right sequence close to the end of the BamHI B fragment (see Fig. 5). After digestion with BamHI no hairpin structure was ob-

Fig. 2. Electron micrographs of heteroduplexes between cpDNA from strain bacillaris and cloned fragments from the same strain and from strain $Z$.

a) A random cpDNA fragment hybridized with two molecules of the plasmid pRBH26 which was linearized with Sall. One plasmid fragment annealed with the second $r \mathrm{rn}$ operon $(\mathrm{rrnB})$ and another one with the third operon $(\mathrm{rrnC})$ as can be identified by the distances from the hairpin and from the stem/loop 13 . The short and the long single-stranded ends of the plasmid are vector sequences. The heteroduplex with the $\operatorname{rrnB}$ operon shows a small deletion loop (del).

b) Heteroduplex between the BamHI fragment $B$ of cpDNA and pRBH26 DNA which was linearized with Sall. The 16S rRNA gene of the rrnA operon of the plasmid DNA has annealed with the extra 16S rRNA gene in the BamHI B fragment, the 3'-end of the 23S rRNA gene with a sequence downstream of the stem/loop 13, and the 5'-end of the 5S rRNA gene with the end of BamHI B, forming a small single-stranded loop visible as a knob.

c) The same heteroduplex structure as in b), but with the fragment BamHI E cut out from the plasmid pRBH26. This shows more clearly the small single-stranded loop situated between the homologies of the $3^{\prime}$-ends of the 23S rRNA genes and the homologies of the 5 -ends of the 5S rRNA genes. This loop corresponds to the right-hand side of the duplicated inverted sequence (see Fig. 5) which forms the hairpin structure in the undigested cpDNA.

d) Heteroduplex between a cpDNA fragment Sacll C from the bacillaris strain and a cloned EcoRI-BamHI fragment from the $Z$-strain. The structures of the two fragments are drawn in the map underneath the tracing. The restriction enzyme Sacll cuts the Euglena cpDNA only within the 16S rRNA gene sequence. Therefore one end of the bacillaris fragment contains the extra 16S rRNA sequence downst ream of the Sacll site and the other end the sequence upstream of the Sacll site of the next I6S rRNA gene of the rrnA operon (see drawing). The $Z$ strain fragment was obtained from a plasmid which contained the whole region from the extra 16S rRNA gene to the EcoR I site within the 23S rRNA gene of the rrnA operon in the vector pBR322 (Roux et al. in prep.). By digesting the plasmid DNA with BamHI a fragment was obtained which contained a short stretch of vector DNA, part of the 23S rRNA gene and the whole 16S rRNA gene of the rrnA operon and most of the sequence between the inverted repeats II. The inverted repeat sequence at the 5'-end was cut off and the single-stranded fragment cannot form a stem loop structure (see drawing). S, B, and E mark the positions of the SacII, BamHI, and EcoRI recognition sites respectively. The hatched region represents a vector sequence.

The single-stranded tail in the heteroduplex is the $3^{\prime}$-end of the $23 \mathrm{~S}$ rRNA gene of the $\mathrm{Z}$ strain fragment. The $16 \mathrm{~S}$ rRNA sequence of this fragment annealed with the two ends of the bacillaris $16 \mathrm{~S}$ rRNA gene, thereby forming a circular structure. The duplex continues with the leader sequence up to the hairpin, with the sequence in the hairpin loop, and with the sequence in the loop formed by the inverted repeat 13. In the tracing the bacillaris fragment is $d r a w n$ as a solid line, and the $Z$ strain fragment as a dashed line. The triangle points to the stem of the hairpin. 
served and the stem/loop structure 13 was found near the end of BamHI B. A heteroduplex between BamHI B and the linearized pRBH26 DNA is shown in Fig. 2b. The shorter single-stranded tail is the end of the plasmid and includes the short vector sequence, the $100 \mathrm{bp}$ of the $5 \mathrm{~S}$ rRNA and most of the 5S-16S spacer. The $16 \mathrm{~S}$ rRNA duplex extends about 250 bp into the leader region. This indicates that the gene could have a functional promotor. Most of the 23S rRNA gene of the BamHI E fragment is not base-paired, but a sequence of about $300 \mathrm{bp}$ of the 3 -end of the gene has annealed with the sequence just downstream of I3. At the ends of the two BamHI fragments a small hook is formed which sometimes opened up to a loop. The long single-stranded tail is pBR322. A similar heteroduplex was prepared with the BamHI E fragment after removing the vector sequences of $\mathrm{pRBH} 26$ (Fig. 2c). This picture shows clearly that the ends of the two BamHI fragments which contain the $26 \mathrm{bp}$ of the 5 -end of the 5S rRNA gene, have annealed, and that the right hand hairpin sequence is folded to a small loop. Theoretically the other end of the BamHI E fragment could anneal with this loop because it contains the complementary left-hand hairpin sequence. Presumably this is prevented by sterical problems.

The stem/loop has a structure which is very similar but not identical with the stem/loop II of the strain $\mathrm{Z}$. The loop in the bacillaris strain has a length of about $0.8 \mathrm{kbp}$ whereas the corresponding loop in strain $\mathrm{Z}$ has a length of $1.2 \mathrm{kbp}$ (11). The stem structure in bacillaris is less stable than in the strain $\mathrm{Z}$ in cytochrome spreadings. This could be due either to a shorter inverted sequence, to base mismatching or to a higher AT-content. To find out whether these regions of the two strains have any common sequences a heteroduplex was prepared which is shown in Fig. 2d. The inverted repeats can prevent the reannealing of the loop sequences even in homoduplexes, because the free movement of the two strands is inhibited. Therefore the $Z$ strain DNA was cut in a way which removed the right hand inverted repeat of $I 1$. The structures of the two fragments used for the heteroduplex are drawn at the bottom right of Fig. 2d. The heteroduplex shows that the 16S rRNA sequence, the leader sequence up to the hairpin, the sequence in the loop of the hairpin, and the loop of $\mathrm{I} 3$ have annealed with the corresponding $\mathrm{Z}$ strain sequences. The stem of the hairpin is not available for annealing with another strand and the corresponding $Z$ strain sequence can be seen as a single-strand running parallel to the hairpin (see tracing). The duplex formed by the loops of the two stem/loop structures shows that 11 and $\mathrm{I3}$, although different, contain homologous sequences. It is possible that the sequences are identical and that only one of the inverted repeats is shorter and placed in a different position.

The cpDNA of the Z-S strain was analysed in the same way as described in Figs. 1 and 2 for the bacillaris strain. Heteroduplexes with the $E$. coli and with pR BH26 DNA showed that this strain has only one complete rrn operon and was described before (21). The region upstream of the complete operon shows the same features as in the bacillaris strain. It includes the extra $16 \mathrm{~S}$ and the extra partial $23 \mathrm{~S}$ rRNA sequence, the hairpin and the stem/loop I3.

For a direct analysis of the two deleted rrn operons and other possible non-homologies, the cpDNAs of both strains were cut with Sall and hybridized with each other. A heteroduplex between the Sall B fragments, which contain all rRNA genes, is shown in Fig. 3a. A large deletion/ substitution loop is formed. The size of the loop was measured as $11.6 \pm 0.7 \mathrm{kbp}$. The size of the BamHI $\mathrm{E}$ fragment was measured (using mica adsorptions) as $5.8 \mathrm{kbp}$. The size difference between the two strains corresponds to the size of two repeated rrn units. The loop was localized at variable positions between 10.8 and $15.5 \mathrm{kbp}$ away from the nearest Sall site, which is downstream of the rrn operon. However, the majority of the loops was clustered at a distance of $12.8 \mathrm{kbp}$ from the end of the fragment. The position of the hairpin was measured in singlestranded SalI B fragments. With these measurements the predominant position of the deletion/ insertion loop was determined about $4 \mathrm{kbp}$ downstream of the hairpin, which is near the middle of the 23S rRNA gene (see Fig. 5).

The small deletion loop indicated in the heteroduplex was also found in homoduplexes of selfannealed DNA from both strains (Fig. $3 b$ and c). These loops have a variable size and appear at a position about $7.4 \mathrm{kbp}$ away from the nearest SalI site upstream of the rrn operons. This position corresponds to the region of variable size, which was found earlier in the $\mathrm{Z}$ strain $(10,18)$ and which mapped close to the origin of replication (14). 

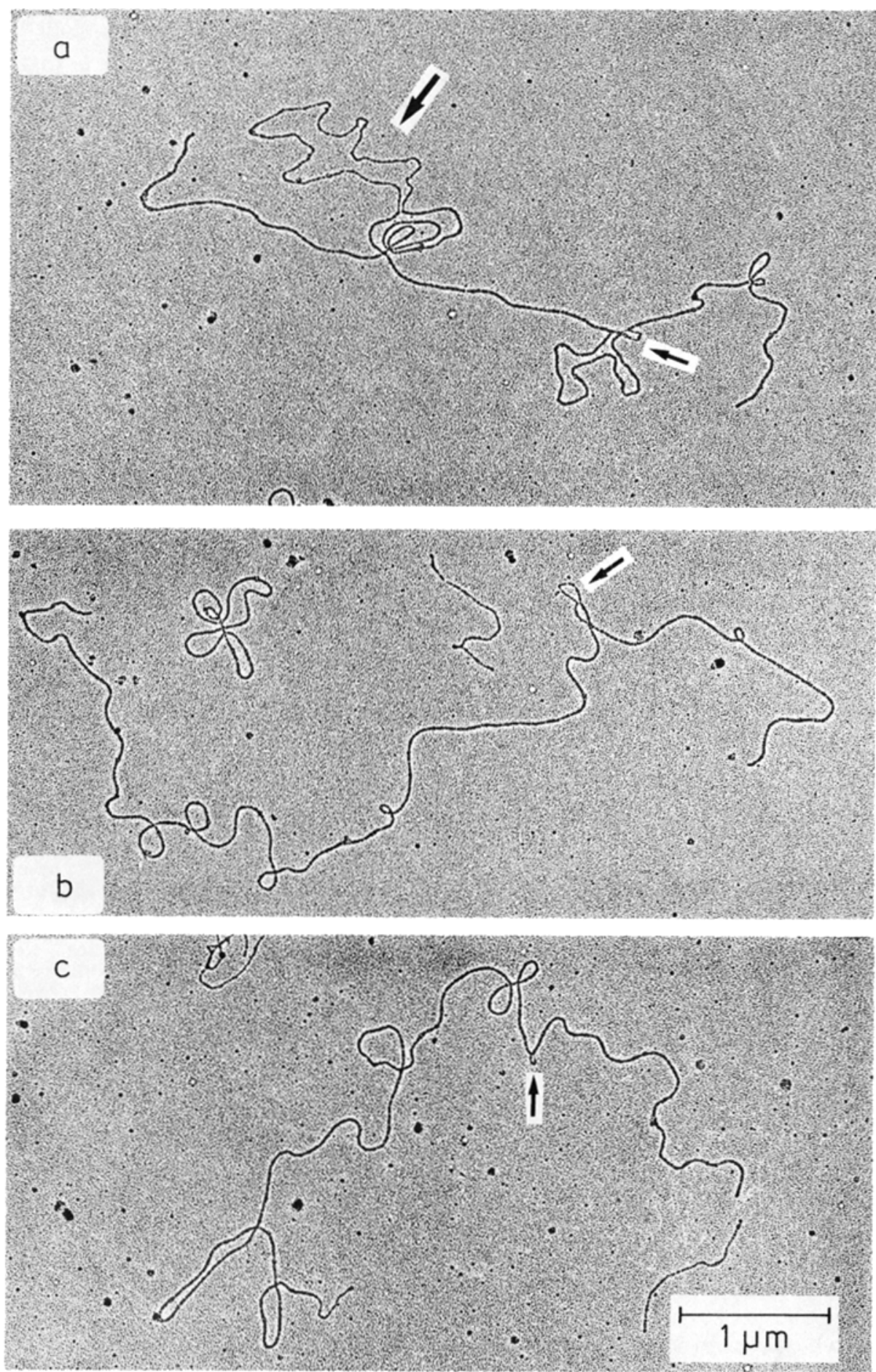

Fig. 3. Electron micrographs of hetero- and homoduplexes of fragments from the bacillaris and Z-S strains.

a) Heteroduplex between the Sall B fragments from strains bacillaris and Z-S. The long single-stranded deletion/insertion loop represents the two rrn operon units of the bacillaris strain (large arrow), which are missing in strain Z-S. The small arrow points to another short single-stranded loop, which can also be found in homoduplexes of both strains.

b) and c) Homoduplexes of self-annealed Sall B fragments of strain Z-S, showing different sizes of the small deletion/insertion loop as in a) at the same position, indicating a region of variable size in otherwise homologous duplex molecules. 


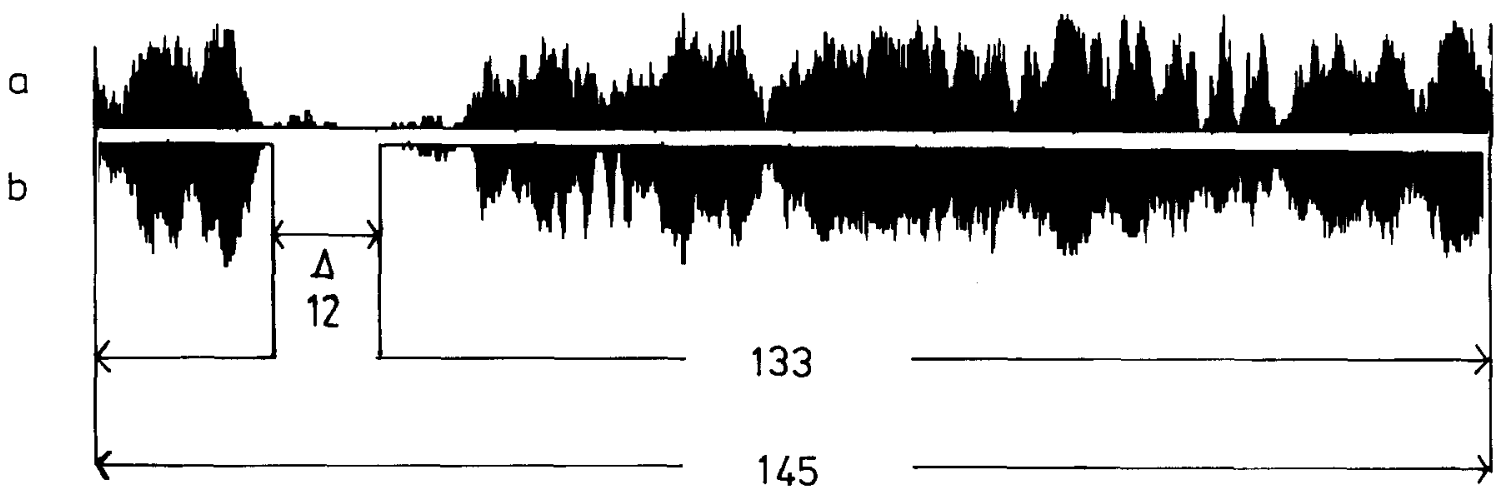

Fig. 4. Partial denaturation histograms from strain bacillaris (a) and from strain Z-S (b). The histograms were derived from the partial denaturation patterns of about 20 circular molecules each, and aligned by circular permutation. The black areas represent singlestranded denatured regions. The long non-denatured regions contain the GC-rich rrn operons. The molecular sizes of the circular cpDNAs were determined from cytochrome spreadings of non-denatured molecules, using T7 DNA as internal length standard (4).

To determine whether there are differences elsewhere in the genomes, the cpDNAs were compared by partial denaturation mapping (Fig. 4). Both maps clearly show the GC-rich regions of the rrn operons. When the map of the Z-S strain is cut and shifted to account for the deletion of the two rRNA operon units, the two patterns can be lined up and match well over the whole genome. It can be concluded that the strains have evolved from each other by one deletion or insertion event, and are otherwise identical. Small rearrangements of less than $1 \mathrm{kbp}$ cannot be ruled out strictly using this method of comparison.

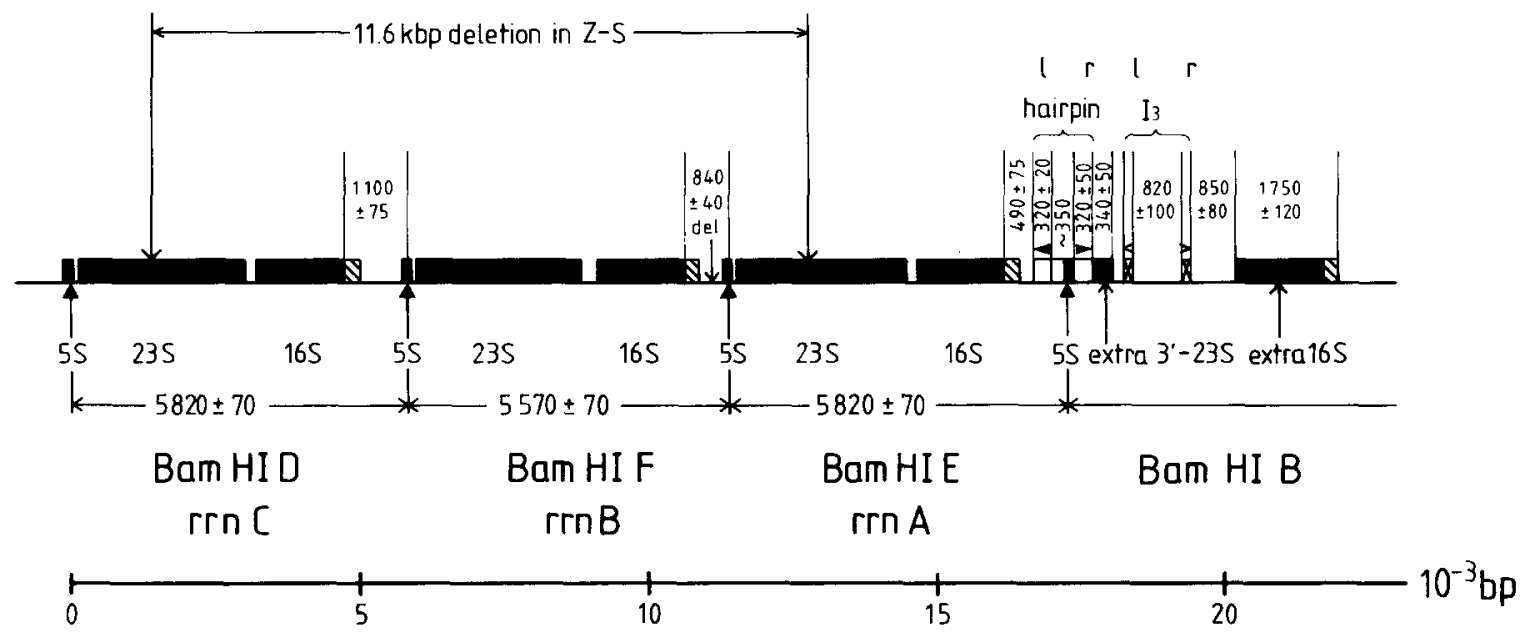

Fig. 5. Schematic drawing of the structure of the rRNA gene region in the bacillaris and Z-S strains. The sizes of the BamHI fragments D, $E$ and $F$ were obtained from measurements of double-stranded fragments adsorbed onto mica, using PM2 DNA as reference. Sequences representing rRNA genes are indicated by black bars. The inverted repeat sequences of I3 are drawn cross-hatched and marked with open arrows. The inverted repeat sequences forming the hairpin structure are marked with short black arrows. The left and right hand sequences of the inverted repeats are marked 'l' and ' $\mathrm{l}$ '. The sequence upstream of the 5'-end of the extra 16S rRNA gene and the homologous sequences in the corresponding positions of the complete rrn operons are marked by diagonal hatching. The position of the two rrn operon units missing in the Z-S strain is derived from the preferred location of the deletion loop found in heteroduplexes as shown in Fig. 3a. However, the position of the loop can vary within the range of the rrn operon unit. Distances are given in basepairs. Values which are given with standard deviations are based on direct measurements. Other values without standard deviation are derived as differences from several measured values. 


\section{Discussion}

The number of rRNA genes in the chloroplast genomes of higher plants is very stable. Most plant species have two rrn operons arranged in an inverted orientation. Very few plants have only one operon (20). In contrast, the number of rrn operons in the chloroplast genomes of Euglena gracilis fluctuates between one in the Z-S strain, three in the Z and the bacillaris strains, and five in the bacillarisATCC strain $(21,16,7,13)$. The tandem arrangement of repeated units seems to facilitate the a mplification or reduction of these units. It is not known whether the number of rrn operons influences the variability of the strains. Under our growth conditions all strains grow at approximately the same rate.

Comparing the structures of the rrn operons and the extra and partial rRNA sequences, the four strains can be devided into two groups. The $\mathrm{Z}$ strain and the bacillaris-ATCC strain form the group of Z-type strains, and the bacillaris and the Z-S strain the group of bacillaris-type strains. The strains within each group seem to have evolved from each other by a deletion or insertion of repeated units. Assuming the Z-S strain originated from the bacillaris strain by a deletion of two rrn operons via unequal crossing-over between the repeated units, the sequences on both sides in the single-stranded deletion loop (Fig. 3a) should be identical with the sequences of the flanking duplex regions. Therefore, theoretically the deletion loop in the heteroduplexes could be expected at any position within the range of the complete rrn operon unit of the Z-S strain. However, the deletion loop was found predominantly within a narrow range in the 23S rRNA gene. This could be explained either by very small, so far unrecognized differences between the 23S rRNA genes or by branch migration during or after the heteroduplex formation which moved the loop to an energetically favoured position.

All four strains have an extra 16S rRNA gene. In the $\mathrm{Z}$ strain a $28 \mathrm{bp}$ inverted repeat was found in the leader region of the extra 16S rRNA gene (17). The $3^{\prime}$-end of the gene is followed by a sequence which is homologous with the sequence downstream of the $5 S$ rRNA gene of the complete operons $(12,17,6)$. The $28 \mathrm{bp}$ inverted duplication in the leader and the additional 5S-16S spacer sequence have not been observed in the bacillaris-type strains. On the other hand, the bacillaris-type strains have an extra partial 23S rRNA sequence and an inverted duplication of part of the $5 \mathrm{~S}-16 \mathrm{~S}$ spacer which is not present in the Z-type strains. Possibly the structure upstream of the complete rrn operons was originally a complete operon which has undergone extensive rearrangements. It remains to be seen whether any of the segments of the 'mutilated' operon are functional and give rise to stable transcripts.

The region characterised by the stem/loop structures $I 1$ and $I 3$ have similar (possibly identical) sequences in all strains. Nothing is known about the function of this sequence. In an analysis of chloroplast DNA-RNA hybrids we found that this region hybridizes with an RNA and may be coding sequence for a protein (unpublished results). Sequence analysis of part of the region revealed an open reading frame (17). Since the sequence is positioned between two repeated 16S rRNA genes it could potentially be deleted by the same mechanism which leads to the deletion of two complete rrn operons in the Z-S strain. The fact that all four strains which we have analysed retained this sequence suggests that it may code for an indispensable gene. The partial denaturation patterns indicate that it is relatively GC-rich and probably is not repeated elsewhere in the genome. It is possible that the insertion of this sequence was involved in producing the rearrangements observed in this region.

The comparison of the two groups of Euglena gracilis strains raises the question about the rate and the mechanism of evolution of these chloroplast genomes. It is believed that the $\mathrm{Z}$ strain has originated in Europe and the bacillaris strain in the United States (15, 1, 6; strains nr. 25 and nr. 15, respectively). Some isolates were listed as early as 1938 but unfortunately it cannot be ruled out that the strains have been mixed up in their long laboratory lives. The results described above demonstrate that the nomenclature of the strains does not necessarily reflect their relatedness on the molecular level. The Z-S strain was thought to be a $\mathrm{Z}$ strain (21), and the bacillaris-ATCC strain was thought to be a bacillaris strain (13). To draw any firm conclusions about the evolutionary relationship of Euglena gracilis strains, it would be necessary to compare the laboratory strains with newly isolated field strains with a well defined origin. 


\section{Acknowledgement}

We thank Jill Clarke for skilled technical assistance.

\section{References}

1. A catalogue of laboratory strains of free-living and parasitic protozoa. J Protozool 5:1-38, 1958.

2. Boros I, Kiss A, Venetianer P: Physical map of seven ribosomal RNA genes of Escherichia coli. Nucleic Acids Res 6:1817, 1979.

3. Delius H, Koller B: Sequence homologies between Escherichia coli and chloroplast ribosomal DNA as seen by heteroduplex analysis. J Mol Biol 142:247-261, 1980.

4. Dunn JJ, Studier FW: Complete nucleotide sequence of bacteriophage T7 DNA and locations of T7 genetic elements. J Mol Biol 166:477-535, 1983.

5. ElGewely MR, Lomax MI, Lau ET, Helling RB, Farmerie W, Barnett WE: A map of specific cleavage sites and tRNA genes in the chloroplast genome of Euglena gracilis bacillaris. Mol Gen Gen 181:296-305, 1981.

6. ElGewely MR, Helling RB, Dibbits JGTh: Sequence and evolution of the region between the rrn operons in the chloroplast genome of Euglena gracilis. Mol Gen Gen, 1983, in press.

7. Helling RB, ElGewely MR, Lomax MI, Baumgartner HE, Schwartzbach SD, Barnett WE: Organization of the chloroplast ribosomal RNA genes of Euglena gracilis bacillaris. Mol gen Gen 174:1-4, 1979.

8. Inman R B, Schnoes M: Partial denaturation of thymine-and 5-bromouracil-containing $\lambda$ DNA in alkali. $J$ Mol Biol 49:93-98, 1970.

9. Jenni B, Stutz E: Analysis of Euglena gracilis chloroplast DNA. Mapping of a DNA sequence complementary to $16 \mathrm{~S}$ rRNA outside of the three rRNA gene sets. FEBS Lett 102:95-99, 1979.
10. Jenni B, Fasnacht M, Stutz E: The multiple copies of Euglena gracilis chloroplast genome are not uniform in size. FEBS Lett 125:175-179, 1981.

11. Koller B, Delius H: Electron microscopic analysis of the extra $16 \mathrm{~S}$ rRNA gene and its neighbourhood in chloroplast DNA from Euglena gracilis strain Z. FEBS Lett 139:86-92, 1982a.

12. Koller B, Delius H: Parts of the sequence between the complete rRNA operons are repeated on either side of the extra 16S rRNA gene in chloroplast DNA of Euglena gracilis strain Z. FEBS Lett 140:198-202, 1982b.

13. Koller B, Delius H: A chloroplast DNA of Euglena gracilis with five complete rRNA operons and two extra 16S rRNA genes. Mol Gen Gen 188:305-308, 1982c.

14. Koller B, Delius H: Origin of replication in chloroplast DNA of Euglena gracilis located close to the region of variable size. The EMBO J 1:995-998, 1982d.

15. Pringsheim EG, Pringsheim O: Experimental Elimination of chromatophores and Eye-spots in Euglena gracilis. The New Phytologist 51:65-75, 1952.

16. Rawson JRY, Kushner SR, Vapnek D, Kirby N, Boerma A, Boerma CL: Chloroplast ribosomal RNA genes in Euglena gracilis exist as three clustered tandem repeats. Gene 3:191-209, 1978.

17. Roux E, Graf L, Stutz E: Nucleotide sequence of a truncated rRNA operon of Euglena gracilis chloroplast genome. Nucleic Acids Res 11:1957-1968, 1983.

18. Schlunegger B, Fasnacht M, Stutz E, Koller B, Delius H: Analysis of a polymorphic region of the Euglena gracilis chloroplast genome. Biochem Biophys Acta 739:114-1231, 1983.

19. Stueber D, Bujard H: Electron microscopy of DNA: Determination of absolute molecular weights and linear density. Mol gen Gen 154:299-303, 1977.

20. Whitfeld PR, Bottomley W: Organization and structure of chloroplast genes. Ann Rev Plant Physiol 34:279-310, 1983

21. Wurtz EA, Buetow DE: Intraspecific variation in the structural organization and redundancy of chloroplast DNA cistrons in Euglena gracilis. Current Genetics 3:181-187, 1981. 